\title{
Dr. Carlos R. Lanfranco La Hoz Semblanza
}

\author{
RAFAEL JARA \\ Profesor Principal de la Faculrad de Medicina, UNMSM
}

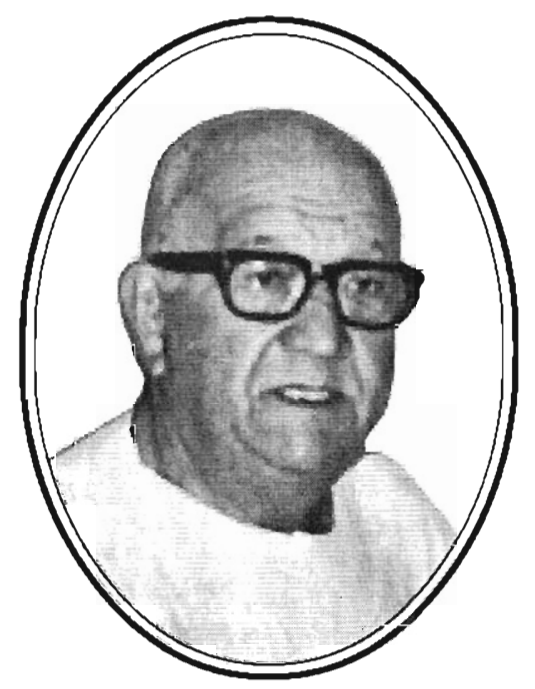

Ha fallecido en Lima el Dr. Carlos R. Lanfranco La Hoz. el maestro más representativo que ha tenido la Facultad de Medicina de San Fernando de la Universidad Nacional Mayor de San Marcos en los últimos cuarenta años. Fue médico y maestro universitario, hombre de ciencia, extraordinario semiólogo, gran clínico, pero sobre todo docente por naturaleza. Gremialista cuando los acontecimientos lo requerían. con gran sensibilidad por el dolor humano y ejemplo de muchas generaciones de médicos.

Nació en Chucuito, Callao, un 28 de Mayo de 1917. hijo primogénito del matrimonio de Carlos Alberto Lanfranco Bernales y la señora Regina La $\mathrm{Hoz}$ Pastrana. Transcurrió su vida muy cerca del Mar de Grau, en Chucuito, en una casa ubicada en la calle Gamarra $N^{\circ}$ 313. Realizó su etapa escolar inicial en el Colegio San José de Clunny y continuó luego sus

\footnotetext{
Correspondencia:

Dr. Rafael Jara Palacios

Servicio Julian Arce

Hospital Nacional Dos de Mayo

Parque de la Medicina. Av. Graus/n. Lima l, Perí
}

estudios en el colegio de los Hermanos Maristas. Muy inquieto en su infancia, solía jugar al fútbol en el puesto de defensa central, pero su gran pasión por la natación lo llevó a integrar un equipo de water polo, logrando representar al Perú en competencias internacionales. Practicó esta disciplina deportiva hasta pocos años antes de su fallecimiento.

Poco antes de finalizar la secundaria inicia su vocación de docente, pues comienza a enseñar el curso de Ciencias Naturales, Fisiología e Higiene, Química Orgánica e Inorgánica, en el Gran Colegio Nacional Dos de Mayo del Callao.

Debido a los problemas políticos surgidos en la época del gobierno del Presidente Augusto B. Leguía, fue clausurada la Universidad Nacional Mayor de San Marcos, lo que obligó al maestro a viajar a Santiago de Chile, obteniendo un año después el bachillerato en Filosofía con mención en Biología y Química.

En 1934, reabierta la Universidad de San Marcos, regresó al país haciendo Pre-Médicas en el año 1936. Pero al producirse un nuevo receso de la Universidad, se vio obligado a regresar a Chile, donde ingresó a la 
Universidad de Concepción. En 1940 retornó al Perú y reingresó a la Facultad de San Fernando al 5to. año de medicina. Meses después, por sus condiciones innatas para la docencia. ingresó como ayudante ad honorem a la cátedra de "Clínica Médica, Nosografía y Terapéutica" que dirigía el profesor Sergio E. Bernales Sánchez.

En 1942 realizó el internado médico en el Hospital Dos de Mayo, optando en 1943, el grado académico de Bachiller en Medicina con la tesis "Infecciones focales y glomerulonefritis difusa, posibles exacerbaciones de ésta por la amigdalectomía”.

En 1943, fue promovido como ayudante rentado y en 1944 nombrado Jefe de Clínicas y simuitáneamente ingresó a la cátedra de "Enfermedades Tropicales Infecciosas y Parasitarias", que dirigía el Dr. Oswaldo Hercelles García.

En 1948, es nombrado Profesor Auxiliar; en 1961 Profesor Asociado y en 1962 promovido a la condición de Profesor Principal por concurso internacional.

A partir de ese año, el profesor Lanfranco comenzó su tarea de apostolado en la enseñanza médica. Llegaba al hospital antes de las ocho de la mañana y se retiraba a las cinco de la tarde, entregándose a sus alumnos con cariño y amor en su gran afán de lograr la formación integral del estudiante de medicina, brindándole la perfecta imagen del paradigma médico.

En 1961 fue miembro de la Junta Transitoria de Administración de la Facultad de Medicina.

Fue miembro del Consejo de Facultad, del Consejo Universitario y de la Asamblea Universitaria, en varias oportunidades. Miembro del ler. Seminario Viajero de Decanos; Delegado ante la Unión Latinoamericana de Facultades de Medicina. También fue miembro fundador del Instituto de Investigaciones Clínicas, demostrando profunda preocupación por que la investigación científica sea una tarea fundamental en la génesis del conocimiento. En el año 1968 fue elegido Decano de la Facultad de Medicina, y renunció al cargo en 1969 por no estar de acuerdo con el nuevo sistema de departamentalización. En 1970 fue elegido por unanimidad Jefe del Departamento de Medicina.

En 1989 fue designado Profesor Emérito de la Universidad Nacional Mayor de San Marcos.
En lo referente a su carrera médico asistencial, entre 1943 a 1945 se incorporó a la Sala Julián Arce de] Hospital Dos de Mayo, que dirigía el Dr. Sergio E. Bernales, como asistente libre encargado de los Censos y las Estadísticas de las Presentaciones Clínicas. Es nombrado en 1946 médico asistente. En 1959, como Jefe Interino de la Sala Julián Arce hasta 1961, accedió por concurso en forma definitiva a dicho cargo en 1962. En 1978 es nombrado Jefe del Departamento de Medicina hasta 1983 y luego de 1985 a 1987.

El Dr. Carlos Lanfranco desarrolló una conducta inteligente en la dirección y elaboración del curso de Medicina Interna, fue el verdadero eje en la formación planificada y minuciosa de los futuros médicos peruanos. Cada detalle del curso era cuidadosamente elaborado junto con sus profesores Jefes de Capítulo, escuchando sus sugerencias que volcaba en el sylabus del curso. Fue el introductor de los capítulos de Toxicología. Reumatología, Metabolismo y Nutrición e Inmunología Básica y Clínica. Conducía los Conversatorios Clínicos y Clínicos Patológicos con verdadera maestría; realizaba las Mesas Redondas con docentes invitados de gran prestigio, motivando la participación de profesores y alumnos, reuniones científicas que se prolongaban sobrepasando largamente el horario establecido, y sin que alguien mostrara signos de cansancio, debido al interés que despertaba estas actividades académicas.

Un buen médico, nos decía, debe tener las siguientes cualidades: "ser sagaz, observador, perspicaz, prolijo, exhaustivo y debe estar capacitado para trabajar 14 horas diarias". También tenía términos o frases muy propios de él, como: "fruto y producto", "es menester e indispensable", "eso lo veremos en su debido momento", "con más fervor". Orra cualidad destacada era su memoria prodigiosa que le permitía llamar a cada alumno por sus nombres y apellidos con sólo haber pasado lista una sola vez, y conocer la Historia Clínica de cada paciente con lujo de detalles.

Quienes tuvimos el privilegio de conocerlo muy de cerca, encontramos en él una persona con carisma excepcional, con una sólida formación moral y humanística, que no solamente transmitía conocimiento y habilidades sino que, de manera especial, fomentaba el cultivo de valores indispensables para el logro de una óptima formación profesional y personal, sustentada en el ejemplo de médico probo con gran sensibilidad por el dolor humano, que defendió ardorosamente sus puntos de vista, sus convicciones 
sin rencores ni revanchismo. Al analizar algo de su pensamiento, podemos dar fe de su profundo conocimiento psicológico acerca de los profesores que lo acompañaban y de los alumnos, motivándolos e inculcándoles su pedagogía y destreza en el examen clínico en la cama del paciente en forma exhaustiva. De esta manera conseguía que los alumnos aprendieran lo universal y medular de la medicina que es la práctica clínica ante la cabecera del enfermo, lo cual lo proyecta como un paradigma de la medicina nacional.

\section{Cargos y participación institucional:}

- Presidente de la Asociación Peruana de Facultades de Medicina (ASPEFAM), 1968-69.

- Presidente de la Academia Nacional de Medicina, 1985-87.

- Presidente de la Asociación Latino Americana de Academias Nacionales de Medicina (ALANAN).

- Presidente del Consejo Consultivo de la Fundación Instituto Hipólito Unanue.

- Presidente de la Asociación de Médicos del Hospital Dos de Mayo.

- Miembro de la Junta Directiva de la Federación Médica Peruana, 1952-58.

- Miembro de la Comisión Consultiva de Salud del Consejo Nacional de Ciencia y Tecnología (CONCYTEC).

- Miembro de múltiples sociedades científicas nacionales e internacionales.

\section{Publicaciones:}

- Tratado de Terapéutica, 1982.
- "La medicina y su desarrollo a partir del descubrimiento de América".

- "Foro sobre educación y salud", coautor.

- "Síntesis biográfica de Daniel A. Carrión".

- Trabajos publicados en revistas científicas nacionales e internacionales sobre diabetes mellitus, hipertensión arterial y enfermedades renales.

\section{Distinciones:}

- Primer Premio Sandoz al mejor trabajo científico, 1983.

- Presidente Honorario, Sociedad Peruana de Medicina Interna.

- Llevan su nombre promociones médicas 1967, 1974, 1975 y 1999, así como la residentes 1977, 1980 y 1996 de la Facultad de Medicina de la Universidad Nacional Mayor de San Marcos.

- La Sociedad Peruana de Medicina Interna instituyó en 1989 el Premio "Carlos Lanfranco La Hoz", para el mejor trabajo científico presentado por residentes.

- Académico Honorario de la Academia Nacional de Medicina, 1996.

\section{Condecoraciones:}

- Condecoración de la Orden de Daniel A. Carrión.

- Medalla de Oro, Fundación Instituto Hipólito Unanue.

- Medalla de Oro, Cuerpo Médico del Hospital Dos de Mayo.

- Medalla de Oro "Alberto Barton".

Lima, 1999. 